\title{
The System Construction of Mathematical Basic Courses for Artificial Intelligence
}

\author{
Wenting Wang ${ }^{1, *}$ \\ ${ }^{1}$ Department of Big Data and Internet, Shenzhen Technology University \\ *Corresponding author. Email:wangwenting@sztu.edu.cn
}

\begin{abstract}
Currently, there is large demand of talents in the area of big data and artificial intelligence talents. Colleges and universities are gradually developing the training system of such talents. Due to the large number of courses in related area with significant difficulty, the situation of mutual reference methods between courses is common. In this paper, the practical education experience is used to research on the establishment of the training system. This paper puts forward the concept of the linkage system between intelligent professional courses and mathematics courses, and expounds the construction methods of the linkage system, in order to achieve the purpose of cultivating undergraduate students and constructing education platform.
\end{abstract}

Keywords: Artificial intelligence, Mathematics, System establishment, Linkage system.

\section{INTRODUCTION}

Since 2015 (the first artificial intelligence conference in China), the concept of "artificial intelligence"(AI) has entered all aspects of social life, such as intelligent manufacturing, intelligent finance, intelligent medical and so on. At present, artificial intelligence has been promoted to the height of national strategy. Since the eighteen Party's Congress, general secretary $\mathrm{Xi}$ Jinping has put innovation in the core position of the overall development of the country, attaches great importance to the development of artificial intelligence, talked about the importance of AI for many times, pointed out the direction for artificial intelligence to empower the new era, and pointed out: "in the next 10 years, it will be the key 10 years for the transformation of new and old energy resources in the world. A new round of scientific and technological revolution and industrial transformation, such as artificial intelligence, big data, quantum information and biotechnology, is gathering strength, giving birth to a large number of new industries, new formats and new models, bringing earth shaking changes to global development and human production and life. We should seize this great opportunity to promote the leapfrog development of emerging market countries and developing countries. "(On July 25, 2018, Xi Jinping's speech at the BRICs business forum.)
How to make effective curriculum training program for undergraduates is the key to the training of artificial intelligence talents. The exploration of basic mathematics course for professional courses is a valuable exploration and attempt to cultivate talents, especially undergraduate students in the background of intelligent era. The author of this paper has participated in the construction of the undergraduate course with artificial intelligence characteristics in the University. In practice, it is found that the purpose of the training platform is to make students have the ability of analyzing, designing and realizing engineering problems in the field of artificial intelligence through theoretical study and practice; Master basic knowledge of mathematics and natural science, master basic theory of computer science, application knowledge of computer software / hardware system, basic theory and core technology, support technology, system platform and application knowledge of artificial intelligence, and be able to analyze and make decisions or enhance human ability according to complex input such as large scale model data in specific fields, It lays a solid foundation for the development of AI system with specific problems.

In order to carry out the cultivation purpose of the specialty class of artificial intelligence, the basic mathematics education problems in the artificial intelligence course explored in this paper, thus forming the system of the combination of artificial intelligence 
course and mathematics course. According to the concept of the system, the professional courses in the course setting, such as introduction to artificial intelligence, optimization method, principle and technology of big data processing, introduction to machine learning, in-depth learning and application, are designed to make undergraduates understand the concept of AI Master the basic principle of intelligent processing and analysis methods and be able to use these methods to carry out the primary artificial intelligence application. All of these courses involve the basic knowledge and calculation methods of mathematics of different categories. The author of this paper, as the current mathematics teacher in the University and the machine learning course teacher in the artificial intelligence class, intends to investigate the mathematics knowledge and the corresponding time required in the professional courses, explore the intersection between the two courses and the basic mathematics courses of undergraduate (higher mathematics, linear algebra, probability theory and Mathematical Statistics), and design an effective knowledge point module, It is used for the introduction of the basic knowledge in the early stage of the professional course or the application of the basic mathematics course, so as to connect the mathematics course with the professional course, and expand the students' knowledge and comprehensive mathematical quality.

Meanwhile, in the course of curriculum construction, we can accumulate teaching experience, collect teaching resources, share the experience and achievements of this course construction, so that more beginners interested in artificial intelligence can benefit from the achievements of this course construction.

\section{BACKGROUND RESEARCH}

The Ministry of education has recently issued the "Artificial Intelligence Innovation Plan of Colleges and Universities", and put forward the goal of "improving the talent training system in the field of artificial intelligence".

In recent ten years, the achievements of Chinese researchers in the fields of artificial intelligence and machine learning have been remarkable. As far as the number of international journals published, they have surpassed the United States and ranked first in the world. However, due to the short development time and insufficient research foundation, the theory and works proposed by Chinese scientists cannot be recognized as the mainstream in the theoretical circle. At present, the general theories, algorithms, models, such as neural network theory, are mostly proposed by mature countries in this field, such as Canada. In addition, the lack of original and innovative ability is another problem that our country's intelligent talents need to face. When these problems are implemented in the basic education work, the following points can be reflected:

\section{(1) The construction of teaching system}

Artificial intelligence has the attribute of interdisciplinary. Its development originated from the simulation of human activities in the industry, and became the focus of industry and research field in the third development of deep learning in 2006. Among them, the research field makes great progress in the related technology of artificial intelligence by using their own basic knowledge, but it also causes the discipline has no natural knowledge system and clear knowledge demand. From the demand of industry, many companies only need the operators at the application level, which can be completed through short-term training, but the following research and development capacity is insufficient, and it cannot provide ideas for the talent training of colleges and universities.

(2) The construction of subject content

In order to improve the original ability of artificial intelligence industry in China, the training of talents should be strengthened from basic knowledge to application ability. Artificial intelligence is to simulate complex functions by neural network structure. The importance of mathematics is especially important in all kinds of basic knowledge. Besides the three basic mathematics of undergraduate course in University: Advanced Mathematics, linear algebra, probability theory and mathematical statistics, there are also many subjects such as mathematical analysis, optimization theory, statistical theory and so on, which are closely related to artificial intelligence. Only by mastering the mathematical principle can we use the generalized perspective to analyse or build a reasonable artificial intelligence model, and know what can be improved and how to improve in the research.

In addition, compared with other majors, AI has the characteristics of domain, that is, there are many disciplines involved, more steps in research and high requirements for talents synthesis. According to the demand of model algorithm calculation force in artificial intelligence application, except mathematics, talents also need to have theoretical and practical requirements in basic knowledge, programming operation, software and hardware platform interaction. The development of artificial intelligence is also very fast. So when developing training plan, we should integrate the ability of questioning, design, modelling, experiment and optimization according to the ability literacy and module, so that it can adapt to even the talent demand in five years.

(3) The construction of the faculty

In 2017, the State Council Circular on printing and distributing the development plan of the new generation 
of artificial intelligence proposed that the discipline of artificial intelligence should be built. We will improve the discipline layout in the field of artificial intelligence, establish artificial intelligence specialty, and promote the construction of the first-class discipline in the field of artificial intelligence. Ma luting, director of the high classroom of the education development research center of the Ministry of education, pointed out that the firstclass discipline itself is the knowledge system, and it is also a system arrangement. When a professional direction is important to a certain extent, the establishment of artificial intelligence discipline at the national level means that there will be more funds and papers in this field, attracting more excellent talents to participate in the academic community. Meanwhile, the talent training scattered in various departments will be more systematic, which will promote the country to take the lead in the world in the new wave of science and technology.

Since the announcement of the State Council on printing and distributing the development plan of the new generation of artificial intelligence, the research of artificial intelligence has been paid unprecedented attention. The National Natural Science Foundation Committee has added the code of artificial intelligence discipline, which sets artificial intelligence in parallel with computer and automation, which promotes the application of related subjects and the gathering of talents.

At present, many universities, research institutes and enterprise units have established many "artificial intelligence colleges" or "big data colleges" to train artificial intelligence talents. After detailed field communication and investigation, we found that there is no artificial intelligence talent training plan and curriculum system widely recognized by the education and industry. At present, the general AI skills are mostly based on mathematical knowledge [1,4], but in the course setting process, it is impossible to effectively combine mathematics with artificial intelligence algorithm and other courses [6-8]. Taking the Institute of artificial intelligence of Nanjing University as an example, in the first semester of 2018-2019 academic year, mathematics courses mainly include mathematical analysis, advanced mathematics and discrete mathematics, while the courses of algorithm and programming mainly include the basis of programming and the introduction of artificial intelligence, There are two problems: (1) there are no pre and post related arrangements between mathematics course and algorithm Course in time and schedule, which often makes the mathematics knowledge involved in algorithm Course lag behind the algorithm course(2) Mathematics course and algorithm course are not effectively connected, so students cannot find the appropriate methodology in the extensive mathematics knowledge to support their study of artificial intelligence major courses. In view of this, this study intends to develop a new education model, which combines the core courses in the process of artificial intelligence talent training organically [8-10].

In addition, although there are many books on artificial intelligence in the market, there are few books for professional talents training, especially for undergraduates. We classify the popular AI books in the market, including science popularization and specialty. The former is used to popularize the concept of artificial intelligence to the public, and the latter is suitable for the research and development of artificial intelligence for professionals. Both of them cannot guide students to carry out comprehensive and solid professional learning from the theoretical basis.

\section{THE SYSTEM CONSTRUCTION OF MATHEMATICAL BASIC COURSES FOR} AI

\subsection{Definition of Core Concepts}

This research puts forward the "continuous system of intelligent course and mathematics course", based on the training of artificial intelligence talents, mining mathematical knowledge points from intelligent courses, re planning the two kinds of disciplines from teaching time, making mathematics serve professional courses, and let students apply mathematics theory knowledge in professional courses, so as to promote the mastery of the two kinds of knowledge, A connection has occurred.

Because the implementation of this paper is the first time to offer Renqin AI course to undergraduates of Shenzhen University of technology, we have not much experience to learn from. Therefore, the main contents of the basic mathematics construction oriented to artificial intelligence constructed in this paper are the summary of the applicant in the past teaching process, or the published academic papers or the stage progress in research. We organize and summarize these research results to form the main construction contents to complete the course construction and teaching tasks with high quality, the construction content proposed by the paper group includes the following aspects:

1. The paper summarizes the mathematics knowledge points and teaching time points involved in the current AI courses, and finds the intersection of relevant knowledge points and basic mathematics courses. For example, the image and photo processing in AI course involves the process of pixel matrix, which requires students to have a cognition of matrix and related operation in linear algebra course; The simple classification in machine learning can use linear regression model in statistics to operate data. 
2. Design knowledge point module which is suitable for undergraduate learning, which can strengthen the knowledge of theoretical course and make a case study; It cannot be too simple and direct, and it cannot be too difficult. The knowledge module can reflect the learning level of most learners. These knowledge modules can be used in the preparation of professional courses or the application case of basic mathematics courses.

3. Prepare the teaching plan of basic mathematics oriented to artificial intelligence, which enables the teaching experience and achievements of this course to settle in the form of textbooks, and make more big data learners benefit from it, and train big data professionals for the national AI strategy.

4. Cultivate the mathematical perception concept of the beginners and practitioners of artificial intelligence, so that they can make better use of the mathematical perception in the future study and work to carry out deep mining and efficient processing of data, and improve their scientific research literacy and employment competitiveness in the field of intelligence.

\subsection{Curriculum Construction Objectives}

The course construction aims at implementing the training purpose of Renqin AI class, based on mathematical perception and from the point of view of "data computability", it aims to cultivate the undergraduates with primary scientific research quality, so that they can understand the concept of AI deeply Master the basic principle of processing and analysis methods and be able to use these methods for primary intelligent application. Meanwhile, in the course of curriculum construction, we can accumulate teaching experience, collect teaching resources, share the experience and achievements of this course construction, so that more beginners interested in big data can benefit from the achievements of this course construction.

\subsection{Research Methods}

Artificial intelligence is a new subject in the scope of university education, different from the traditional mathematics subject "from learning to produce". In the early development of the discipline, it has the characteristics of "production and learning and development" or "from learning and production". That is, the demand and driving force of discipline development are provided by industry, and the knowledge field fills in knowledge base and expands algorithm. Therefore, the artificial intelligence education system in the world has not been developed and perfected. China has the advantage of big data acquisition, and brings more opportunities and challenges to the artificial intelligence industry. It requires high data processing ability and comprehensive analysis ability of talents.

The innovation points of this study are as follows:

1. For the first time, the mathematics knowledge points in the artificial intelligence professional course are compared with the basic mathematics courses, and the intersection is specially used as the research object, and the exploration is carried out from the content and time arrangement;

2. The paper puts forward the "modular knowledge point", clarifies the arrangement method of knowledge in professional and basic courses, facilitates students' classroom learning and self-study, and connects the two necessary skills through "knowledge module", which further improves the comprehensive ability;

3. Refining more perfect teaching process and content from classroom teaching, and promoting content productively in the form of courseware or books, has become the contribution of Shenzhen University of technology to the development of the subject;

4. In combination with the current needs of scientific research and industry, students can be trained accurately, which enables them to have knowledge and learning ability. They can face a variety of industries according to their own advantages and interests: such as Data Engineer, algorithm engineer, scientific research personnel, etc.

\section{CONCLUSION}

At present, the effective cultivation of artificial intelligence and machine learning talents has become an urgent problem. This paper discusses the expected results of the research on the curriculum construction of "intelligent curriculum and mathematics curriculum linkage system facing the forefront of artificial intelligence". It includes the following aspects: the proposal of syllabus, the writing of teaching plan and courseware, the construction of curriculum platform, etc. It is hoped that in the next few years, under the guidance of relevant policies at all levels of the state, province, city and University, the system construction proposed in this paper will be improved and excellent undergraduate graduates will be cultivated.

\section{ACKNOWLEDGMENTS}

The paper is supported by Wenting Wang 's 2020 education reform project of Shenzhen Technology University - Exploration of basic mathematics teaching for artificial intelligence course - 202018660601010.

\section{REFERENCES}

[1] Jia Jiyou. Discussion on the latest hot issues in the application of artificial intelligence in education 
abroad [J]. China audio visual education, 2010 (07): 119-124.

[2] Zhao Jianhua. Overview of intelligent teaching system [J]. China audio visual education, 2007 (07): 10-17.

[3] Zheng Wei Dong, Jiang Geng Hao. An implementation technology of forward reasoning mechanism based on esta [J]. Intelligent computer and application, 2012, 002 (002): p.73-75.

[4] Gao Xiuzhi. Research on mixed teaching mode of higher mathematics. Intelligence, 2016, 36:8.

[5] Yu Wensen, Shi Guimin, Guo Lei, et al. Design and practice of mixed teaching mode for computer elective courses. Education and teaching forum, 2016, 52:268-272.

[6] Zhang Yanqin, pan Liqiang. Research on teaching reform of Higher Vocational Computer Basic Course Based on hybrid teaching. Science and technology of RTVU, 2017, 04:47-48.

[7] Liu Xi, Zhang Suhong. The application of "three in one" mixed teaching mode in talent cultivation of application-oriented universities. Journal of Jinling University of science and Technology (SOCIAL SCIENCE EDITION), 2018, 32 (04): 75-78.

[8] Wan Kun, Yang Qinchuan. An Empirical Study on learners' knowledge construction in blended teaching. Journal of Shangrao Normal University, 2018, 38 (06): 29-35.

[9] Zhang Ruimin, Zhou Tao, Li Shuan Ming, et al. Practice of Hybrid Teaching Reform of computer network and communication course with the goal of ability training. Modernization of education, 2019, 6 (A5): 95-97.

[10] Zuo Jinping. Research and practice of mixed teaching mode in software engineering course. Computer and information technology, 2020, 28 (02): 58-60.

[11] Zhao Lingfeng, Yang Jianbing, Deng Jiong, et al. A preliminary study on the reform of hybrid teaching mode based on OBE concept. Chinese Journal of multimedia and network teaching, 2020, 05:21-23. 